\title{
Pruebas de fusión auditiva y de detección de gaps: Evaluación de la resolución auditiva temporal
}

\section{Auditory fusion and gap detection tests: Assessment of auditory temporal resolution}

\author{
Anthony Marcotti F¹, Bernardita Alvear V'1.
}

\begin{abstract}
RESUMEN
Dentro de los distintos mecanismos del procesamiento auditivo, los aspectos temporales de la audición se consideran como uno de los más relevantes ya que serían la base para el adecuado funcionamiento del resto de los procesos auditivos centrales. Los aspectos temporales de la audición se definen como los procesos implicados en la percepción del sonido y de sus variaciones en el dominio temporal. Uno de los aspectos temporales más ampliamente estudiados, por su relevancia en la percepción y discriminación de los sonidos del habla, ha sido la resolución auditiva temporal, que se refiere a la mínima cantidad de tiempo necesaria para que un individuo pueda discriminar entre dos estímulos auditivos distintos. En los últimos años, se ha utilizado principalmente metodologías de detección de fusión auditiva y detección de gaps para su evaluación. Ambas han dado origen a una serie de pruebas psicoacústicas, de las cuales destacan tres: la prueba de fusión auditiva revisada, la prueba de detección de gaps aleatorios y la prueba de gaps en ruido. Se recomienda la incorporación de alguna de estas pruebas en la evaluación clínica ante la sospecha de trastornos del procesamiento auditivo.
\end{abstract}

Palabras clave: Aspectos temporales de la audición, resolución temporal, detección de gaps, fusión auditiva.

\begin{abstract}
Among the different mechanisms of auditory processing, the temporal aspects of hearing are considered as one of the most relevant since they would be the basis for the proper functioning of the rest of the central auditory processes. Temporal aspects of hearing are defined as the processes involved in the perception of sound and its variations in the temporal domain. One of the most widely studied temporal aspects due to its relevance in the perception and discrimination of speech sounds has been the temporal auditory resolution, which refers to the minimum amount of time necessary for an individual to discriminate between two different auditory stimuli. In recent years, the mainly used methodologies for evaluate it are the hearing fusion detection and gaps detection. Both methodologies have given rise to several psychoacoustic tests, of which
\end{abstract}

1 Carrera de Fonoaudiología. Facultad de Ciencias de la Salud, Universidad de las Américas, Providencia, Chile.

Los autores declaran no tener conflictos de interés.

Recibido el 16 de julio, 2018. Aceptado el 17 de septiembre, 2018. 
three have been highlighted: the revised auditory fusion test, the random gap detection test and gaps in noise test. It is recommended the incorporation of any of these tests in the clinical evaluation of suspected auditory processing disorders.

Key words: Temporal aspects of audition, temporal resolution, gaps detection, auditory fusion.

\section{INTRODUCCIÓN}

En el año 2005, la Asociación Americana de Habla, Lenguaje y Audición (ASHA) publicó el documento más reciente en relación con el procesamiento auditivo (central) o $\mathrm{PA}(\mathrm{C})$ y sus alteraciones ${ }^{1}$. En dicho documento, se definió el $P A(C)$ como la eficiencia y eficacia con que el sistema nervioso central (SNC) utiliza la información auditiva'. De manera más precisa, se ha definido como el procesamiento perceptual de la información auditiva en el SNC y la actividad neurobiológica en el cual se sustenta dicho procesamiento. Tanto la ASHA $^{1}$ como otros autores ${ }^{2,3}$ concuerdan en que el $\mathrm{PA}(\mathrm{C})$ incluye los mecanismos auditivos que sustentan las siguientes habilidades: Iocalización y lateralización sonora, discriminación auditiva, reconocimiento de patrones auditivos, aspectos temporales de la audición, desempeño auditivo con señales acústicas competitivas y desempeño auditivo con señales acústicas degradadas. De estos últimos, los aspectos temporales de la audición son considerados como los más importantes, ya que prácticamente todo el resto de las habilidades dependerían, en mayor o menor medida, de su adecuado funcionamiento.

Los aspectos temporales de la audición son todos aquellos mecanismos implicados en la percep- ción del sonido y de sus variaciones en un dominio temporal restringido 0 bien delimitado ${ }^{4}$. En esta definición se incluyen los siguientes procesos: ordenamiento o secuenciación temporal, resolución o discriminación temporal, integración o sumación temporal y enmascaramiento temporal (Tabla 1).

A pesar de que los distintos aspectos han sido ampliamente estudiados, existe poca claridad en relación con las aplicaciones clínicas que podrían tener los hallazgos en esta materia. En la actualidad, solo existen medidas conductuales clínicamente viables de procesamiento temporal, limitadas al ordenamiento y a la resolución temporal. Aunque, hay una serie de paradigmas informados para la evaluación de la integración y el enmascaramiento temporal, no han sido viables clínicamente debido a las necesidades de equipamiento, condicionamiento de los sujetos evaluados y a los tiempos requeridos para la evaluación ${ }^{4}$. El presente artículo tiene como objetivo presentar una revisión actualizada sobre la importancia de la resolución auditiva temporal y las pruebas clínicas disponibles para su evaluación.

\section{Resolución auditiva temporal}

La resolución auditiva temporal, también llamada discriminación auditiva temporal, se refiere a la

Tabla 1. Aspectos temporales de la audición

\begin{tabular}{|ll|}
\hline Aspecto & Definición \\
\hline Ordenamiento temporal & $\begin{array}{l}\text { Procesamiento de dos o más estímulos auditivos según su orden de aparición u ocurrencia en el } \\
\text { dominio temporal. }\end{array}$ \\
Resolución temporal & Mínimo intervalo de tiempo en el cual un individuo puede discriminar entre dos señales auditivas. \\
Integración temporal & $\begin{array}{l}\text { Sumación de la actividad neuronal en función de la duración de un estímulo acústico y que produce } \\
\text { una mejora en los umbrales auditivos. }\end{array}$ \\
Enmascaramiento temporal & $\begin{array}{l}\text { Presentación no simultánea entre una señal objetivo y un ruido enmascarante, pudiendo este último } \\
\text { aparecer antes (enmascaramiento anterógrado) o después (enmascaramiento retrógrado) de la señal. }\end{array}$ \\
\hline
\end{tabular}

Definiciones extraídas de Musiek \& Chermak' 
mínima cantidad de tiempo necesario para que un individuo pueda discriminar entre dos señales auditivas ${ }^{6}$. Este intervalo de tiempo recibe el nombre de umbral de resolución temporal, agudeza auditiva temporal o tiempo mínimo de integración auditiva ${ }^{7}$, y para la mayoría de las personas, es de alrededor de 2 a $3 \mathrm{~ms}^{8}$. La resolución auditiva temporal ha demostrado ser una capacidad en extremo importante para la comprensión del habla ya que, al procesar este tipo de señales, el sistema auditivo requiere discriminar pequeñas diferencias de tiempo, que pueden ser cambios en la envolvente o cambios en la amplitud modulada de la señal. Se ha planteado incluso que la resolución auditiva temporal constituye un requisito previo para varias habilidades lingüísticas, incluyendo las habilidades de lectoescritura9.

Un ejemplo de la importancia de la resolución auditiva es su participación en la discriminación de algunas claves lingüísticas sutiles necesarias para la diferenciación de los fonemas con un mismo punto y modo articulatorio, pero que se diferencian en la vibración de los pliegues vocales ${ }^{10}$. Este sería el caso de la discriminación entre los fonemas /p/-/b/, /t/-/d/ y /k/-/g/, que sería posible gracias la detección del voice onset time (VOT). EI VOT ha sido definido como el intervalo de tiempo entre la liberación de una consonante oclusiva y el inicio de la vibración de los pliegues vocales ${ }^{11}$. Las consonantes oclusivas sonoras del español son /b/, /d/ y /g/, y se caracterizan por tener un VOT más corto en relación con las consonantes oclusivas sordas $/ \mathrm{p} /$, $/ \mathrm{t} / \mathrm{y} / \mathrm{k} /{ }^{12}$. Así, por ejemplo, el valor crítico del VOT en español para discriminar entre consonantes sonoras y sordas se sitúa entre 5 y 10 $\mathrm{ms}^{13}$. Este tipo de procesamiento es conocido en lingüística como percepción categórica del habla, porque el oyente percibe a partir de las categorías "sonoro" (por ejemplo /b/) o "áfono" (por ejemplo /p/) y no de una graduación de tiempo (no se perciben fonemas con características intermedias). De esta manera, el procesamiento subyacente a la percepción categórica del habla depende de una adecuada resolución auditiva temporal.

En Chile, se ha descrito que en los casos en que se pierden los rasgos distintivos de oclusión y sonoridad entre, por ejemplo, los fonemas /p/ y /b/, los oyentes podrían discriminar utilizando solamente la información acerca de las duraciones absolutas, que corresponde al tiempo transcurrido entre el inicio de la consonante hasta el término de la misma ${ }^{14}$. Por otro lado, Soto-Barba ${ }^{15}$ constata que, en el habla susurrada (forma de hablar en la cual no vibran los pliegues vocales y que, por lo tanto, pierde la información de la sonoridad) el rasgo acústico que permitiría diferenciar los fonemas /p/ y /b/ sería el índice relativo de duración de la sílaba (IREDUS), el que expresa la proporción que ocupa la consonante en relación con la sílaba a la que pertenece. En resumidas cuentas, la duración absoluta de la sílaba y el IREDUS, rasgos que efectivamente diferencian en forma significativa a los fonemas $/ \mathrm{p}-\mathrm{t}-\mathrm{k} / \mathrm{de} / \mathrm{b}-\mathrm{d}-\mathrm{g} /{ }^{16}$ dependerían, al igual que el VOT, de una adecuada habilidad del sistema nervioso auditivo central (SNAC) para detectar pequeñas variaciones de la señal auditiva en el dominio temporal.

El método más comúnmente utilizado para evaIuar la resolución auditiva temporal es establecer un umbral de detección de intervalos de silencio 0 gaps. El umbral de detección de gaps (GDT por sus siglas en inglés), es la menor cantidad de tiempo entre dos señales consecutivas que un oyente puede detectar ${ }^{17}$. Otro método que se ha utilizado con frecuencia es el de la búsqueda del umbral de fusión, que corresponde al máximo intervalo de tiempo entre dos señales en las que el oyente advierte la presencia de un solo estímulo. Las tareas de detección de gaps y de fusión son similares, ya que ambas buscan evaluar la resolución temporal; sin embargo, fusión y separación toman diferentes enfoques para alcanzar el objetivo deseado. Los procesos de detección de gaps generalmente requieren que los sujetos escuchen estímulos presentados con intervalos de silencio aleatorios e indiquen cuándo detecten dos estímulos distintos. Las tareas de detección de fusión, por el contrario, requieren que los sujetos indiquen cuándo se fusionen y se perciban dos estímulos distintos como uno solo. A pesar de que desde un punto de vista psicoacústico ambos conceptos pueden parecer similares, su neurofisiología subyacente sería distinta $^{18}$. El umbral de fusión auditiva se encuentra alrededor de los $203 \mathrm{~ms}^{19}$ y de esta forma, es coherente con el umbral de resolución temporal.

En la metodología para evaluar la resolución auditiva temporal que utiliza la detección de gaps, se pueden distinguir dos paradigmas distintos. Por 
un lado, en el paradigma clásico llamado detección de gaps "dentro del canal", tanto el estímulo o marcador que antecede como el que sucede al intervalo de silencio tienen una composición espectral similar $^{20}$. En otras palabras, este paradigma consiste básicamente en insertar un periodo de silencio en medio de un estímulo auditivo invariable (Figura 1).

De esta manera, el grupo neuronal o canal perceptual estimulado antes y después del gap sería exactamente el mismo ${ }^{21}$. La presentación de este estímulo puede ser tanto de manera monótica como diótica. Por otro lado, en el paradigma de detección de gaps llamado "entre canales", el marcador que antecede al gap varía en su composición espectral en relación con el marcador que lo sucede, por lo menos en media octava (Figura 2) 22 . De esta forma, serían distintos grupos neuronales los estimulados antes y después del gap. El paradigma entre canales podría darse tanto en una presentación diótica como monótica, pero con marcadores de composición espectral diferentes. Sin embargo, también es posible producir este tipo de detección de gaps con una presentación dicótica sin necesidad de variar la composición espectral de los estímulos utilizados. Esto se debería a que, a pesar de utilizar un marcador igual o similar, al estimular de manera no simultánea ambos oídos, se estarían activando grupos neuronales distintos, primero en un oído y luego en el otro ${ }^{23}$.

A pesar de que el primer paradigma es el más utilizado en las pruebas psicoacústicas de evaluación de resolución temporal, su utilidad clínica y validez ecológica estaría altamente cuestionada².

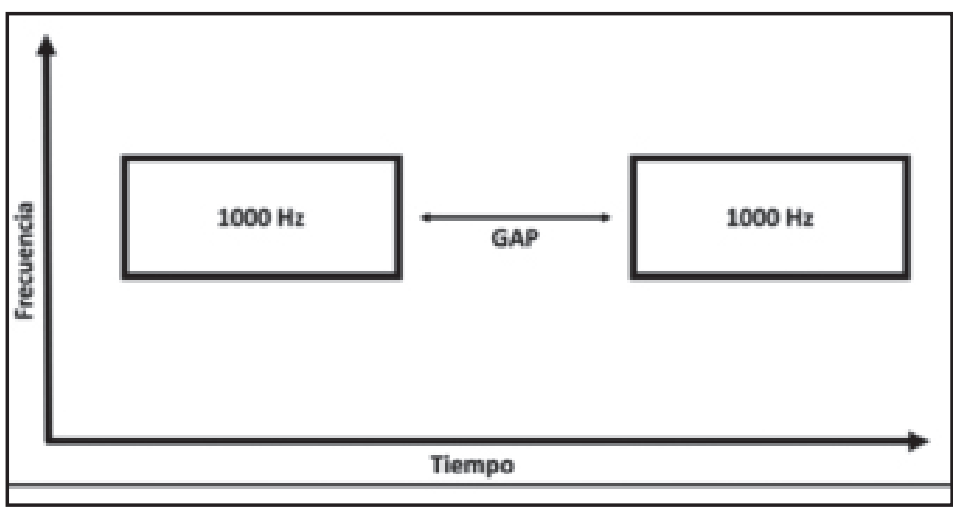

Figura 1. Ejemplo de detección de gaps dentro del canal. Los marcadores o estímulos tienen la misma composición espectral $(1.000 \mathrm{~Hz})$, por lo que se utiliza solo un canal perceptual.

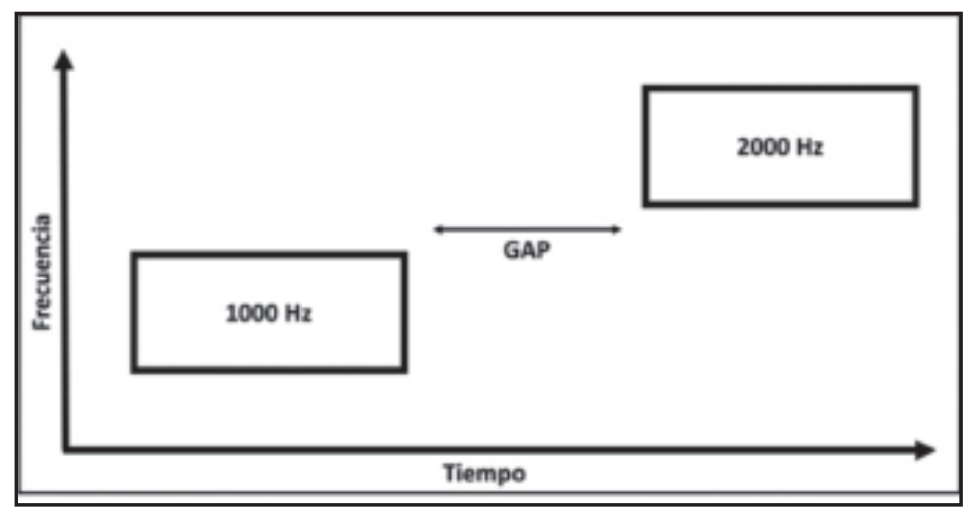

Figura 2. Ejemplo de detección de gaps entre canales. Los marcadores o estímulos tienen distinta composición espectral (1.000 y $2.000 \mathrm{~Hz}$ ), por lo que se utilizan dos canales perceptuales distintos. 
Un ejemplo de esto sería la percepción del VOT durante el habla espontánea, en la cual la composición espectral del fonema oclusivo es distinta a la producida por la posterior vibración de los pliegues vocales y, de esta manera, se estarían utilizando dos canales de procesamiento para acomodar los diferentes tipos de estímulos ${ }^{24}$ : un canal de procesamiento para el primer marcador (fonema oclusivo) y otro para el segundo marcador (vibración de pliegues vocales). Por lo tanto, la gran mayoría de las tareas auditivas cotidianas, incluyendo la percepción del habla, estaría utilizando una modalidad de detección de gaps entre canales y no dentro del canal. Como ya se había mencionado, el paradigma dentro del canal produce un GDT de hasta $2 \mathrm{~ms}$ pudiendo variar hasta $24 \mathrm{~ms}$, mientras que el paradigma entre canales produciría un GDT de entre 14 a $50 \mathrm{~ms}^{25-28}$. Debido a que el GDT es similar cuando se utiliza el paradigma entre canales con presentación dicótica o monoaural, se ha sugerido que estos canales perceptuales podrían estar situados a nivel de SNAC ${ }^{23,25}$. Además, se ha propuesto que el paradigma entre canales tendría acceso a funciones auditivas corticales de orden superior, posiblemente, aprovechando los mismos mecanismos implicados en la percepción del habla ${ }^{24,25}$.

\section{Prueba de fusión auditiva-revisada}

La prueba de fusión auditiva-revisada conocida por sus siglas en ingles AFT-R (de auditory fusion testrevised), fue desarrollada por McCroskey y Keith en el año $1996^{29}$ y es comercializada actualmente por la empresa norteamericana Auditec ${ }^{\circledR}$. Esta prueba tiene una duración aproximada de 10 minutos y consiste en pares de tonos burst, de 15 ms de duración y de frecuencia 0,25, 0,5, 1, 2 y $4 \mathrm{kHz}$. Los pares de estímulos se presentan 18 veces para cada frecuencia en ambos oídos a la vez, con un intervalo de silencio o gap entre ellos que aumentan desde 0 a $40 \mathrm{~ms}$, pasando por 2, 5, 10, 15, 20, 25 y 30 ms (presentación ascendente), y luego disminuyen en duración desde 40 a 0 ms (presentación descendente) pasando también por los intervalos antes mencionados. La presentación de los estímulos se realiza a $50 \mathrm{~dB}$ SL $050 \mathrm{~dB}$ $\mathrm{HL}$ sobre el promedio de los umbrales auditivos aéreos en las frecuencias $0,5,1$ y $2 \mathrm{kHz}$. La prueba se divide en tres subpruebas distintas y no es necesaria su aplicación completa. La subprueba 1 se utiliza como item de práctica y/o screening preliminar; consta de un tono de $0,5 \mathrm{kHz}$ para la calibración del equipo, seguido de la presentación de pares de estímulos a $0,5 \mathrm{kHz}$, con un gap que fluctúa entre 0 y $300 \mathrm{~ms}$. La subprueba 2, también llamada subprueba estándar, se realiza en todas las octavas antes mencionadas, pero esta vez, los gaps fluctúan entre 0 a $40 \mathrm{~ms}$. Finalmente, la subprueba 30 también llamada subprueba extendida, incluye solo tres frecuencias $(0,25,1$ y $4 \mathrm{kHz})$, donde los gaps varían desde 40 a $100 \mathrm{~ms}$, aumentando de a 10 ms y se presentan finalmente dos pares adicionales con un intervalo de presentación interestímulos de 200 y 300 ms.

La tarea del sujeto evaluado es juzgar e indicar verbalmente cuántos sonidos percibe para cada par de estímulos presentados. Si el sujeto logra percibir un gap, y, por lo tanto, logra distinguir dos estímulos distintos, debe verbalizar la palabra "dos". Si el sujeto no logra percibir un gap, y, por ende, solo distingue la presencia de un estímulo, debe verbalizar la palabra "uno". Existen además otras modalidades de respuestas no verbales que pueden resultar convenientes para población pediátrica. Los sujetos evaluados pueden señalar dibujos o tarjetas de respuesta con figuras que representen la cantidad de sonidos escuchados o, simplemente, pueden levantar uno o dos dedos dependiendo de la respuesta deseada. En el ensayo ascendente, el punto de fusión auditiva se identifica como el intervalo de silencio o gap que produce la última respuesta "uno" antes de las respuestas "dos". En el ensayo descendente, el punto de fusión auditiva corresponde al gap que produce la primera de dos respuestas consecutivas "uno". El promedio de los puntos de fusión auditiva ascendente y descendente se conoce como umbral de fusión auditiva para cada una de las frecuencias estudiadas. Si el oyente no logra percibir ningún gap y, por ende, solamente logra identificar un solo estímulo hasta los $40 \mathrm{~ms}$, se puede administrar la subprueba 3 extendida. Los valores normativos de esta prueba han sido obtenidos de manera binaural ${ }^{29}$, sin embargo, más recientemente algunos autores han obtenido algunos valores referenciales para cada oído de manera independiente ${ }^{30}$. Aunque esta prueba fue comercializada recién en el año 1996, los estudios iniciales se llevaron a cabo en 
el año 1980 en la universidad norteamericana de Wichita ${ }^{31}$. Se realizó un estudio en 135 niños de entre 7 a 9 años divididos en tres grupos: un grupo de menores con desarrollo típico, uno de menores con trastornos de lectura y otro con problemas de aprendizaje. A estos menores se les administró la versión original de la prueba de fusión auditiva, llamada en ese entonces Wichita auditory fusion test (WAFT) para realizar una comparación entre grupos. Se describió que los menores con trastornos de lectura y problemas de aprendizaje obtuvieron déficits de resolución temporal, presentando diferencias significativas en los umbrales de fusión auditiva en relación con los niños con desarrollo típico. Sin embargo, entre los grupos de menores con alteraciones de la lectura y de aprendizaje, no se encontraron diferencias significativas, I0 que llevó a los autores de estos estudios a dos conclusiones. En primer lugar, se concluyó que probablemente las alteraciones en el procesamiento auditivo temporal serían la base de algunas alteraciones de lectoescritura y de aprendizaje en general. En segundo lugar, se concluyó que los umbrales de fusión auditiva serían una herramienta efectiva para identificar a sujetos, incluyendo niños, con algún tipo de alteración en las habilidades de resolución temporal. La única diferencia entre la prueba original de 1980 y la comercializada a partir del año 1996, es que esta última se grabó en formato de compact disc para evitar la pérdida de calidad y precisión de los estímulos utilizados en relación con la versión antigua que fue grabada en formato de casete de cinta magnética ${ }^{29}$. A pesar de la existencia de estudios en relación con valores normativos y a estudios que demuestran su alta correlación con otras pruebas de resolución temporal, el AFT-R no cuenta con estudios que documenten su sensibilidad ni especificidad ${ }^{32}$.

\section{Prueba de detección de gaps aleatorios}

La prueba de detección de gaps aleatorios 0 conocida por sus siglas inglés RGDT (de random gap detection test), es quizás una de las pruebas comerciales más ampliamente utilizadas, también comercializada por la empresa Auditec ${ }^{\circledR}$. Esta prueba corresponde a una versión modificada de la AFT- ${ }^{18}$ que, a diferencia de su antecesora y como su nombre lo indica, utiliza el paradigma de detección de gaps. El RGDT consta de cinco subpruebas y utiliza pares de estímulos clics (ruido blanco) de 1 ms de duración y tonos burst de frecuencias $0,5,1$, 2 y $4 \mathrm{kHz}$ de $15 \mathrm{~ms}$ de duración que se presentan en ambos oídos a la vez. Los gaps varían de 0 , $2,5,10,15,20,25,30,35$ y $40 \mathrm{~ms}$ en un orden aleatorio. La subprueba 1 corresponde a un item de práctica para estímulos tonales, compuesta de nueve pares de tonos de $0,5 \mathrm{kHz}$ presentados con intervalos de silencio ascendentes. La subprueba 2 consta de nueve pares de estímulos tonales para cada una de las cuatro frecuencias entre 0,5 y 4 $\mathrm{kHz}$ con gaps presentados de manera aleatoria. $\mathrm{La}$ secuencia de prueba comienza con la frecuencia $0,5 \mathrm{kHz}$, seguido por 1,2 y $4 \mathrm{kHz}$. La subprueba 3 corresponde a un item de práctica para estímulos clic compuesto de nueve pares de clics presentados con gaps ascendente. La subprueba 4 presenta nueve pares de clics separados por gaps con las mismas duraciones de las subpruebas anteriores de manera aleatoria. Existe además una subprueba 5 , que vuelve a presentar pares de estímulos tonales entre 0,5 y $4 \mathrm{kHz}$, pero esta vez con gaps de 50 , $60,70,80,90,100,150,200,250$ y 300 ms. Esta última subprueba no es de aplicación obligatoria, quedando a criterio del evaluador aplicarla en aquellos casos en que el desempeño del sujeto evaluado haya sido insatisfactorio. En contraste con la AFT-R, cada subprueba del RGDT se presenta en condición binaural y a una intensidad de $55 \mathrm{~dB} \mathrm{HL}^{4}$.

Al igual que en el AFT-R, la tarea del sujeto evaluado es juzgar e indicar, de manera verbal, cuantos sonidos percibe para cada par de estímulos presentados. En un comienzo, el oyente debe indicar si percibe "uno" o "dos" estímulos, sin embargo, también se pueden aceptar modalidades de respuesta no verbal, como señalar dibujos 0 figuras. La administración de la prueba requiere alrededor de 10 minutos. Desafortunadamente, sólo hay una lista disponible, lo que limita la capacidad de volver a evaluar a los pacientes, al menos en el corto plazo. El GDT se define como el gap o intervalo de silencio más pequeño que el sujeto pueda identificar como dos estímulos distintos dentro de una frecuencia dada (o el gap más pequeño que conduce a un informe de sujeto de dos clics distintos). Se debe calcular, por último, el umbral RGDT con el promedio de los GDT de las cuatro frecuencias de la subprueba 2. 
A pesar de que el RGDT ya es considerada como una prueba de relativamente rápida aplicación, es posible acortarla aplicando las subpruebas 1 y 2, debido a que sólo los RGT tonales están incluidos en el cálculo del umbral RDGT. Bajo ciertas circunstancias de tiempo que imposibiliten la aplicación de las dos primeras subpruebas completas, el procedimiento podría acortarse aún más, siendo necesario en última instancia, la evaluación de una sola frecuencia de la subprueba 2. Lo anterior debido a que no se han encontrado diferencias significativas en el rendimiento obtenido entre las cuatro frecuencias que utiliza la prueba además de una alta y significativa correlación entre ellas ${ }^{33,34}$. Sin embargo, las pruebas en más de una frecuencia pueden proporcionar información valiosa sobre otras capacidades cognitivas. Así, podría ser una forma indirecta de documentar un problema de atención en algunos niños, incluso si el objetivo de la evaluación con el RGDT es medir la capacidad de resolución auditiva.

En la actualidad, el RGDT cuenta con valores normativos para niños, adultos y adultos mayores, y se encuentran disponibles en la documentación oficial de la prueba ${ }^{18}$. Según Bellis², la información reunida a través varios centros y clínicas en los Estados Unidos parece indicar que un GDT mayor a 20 ms se deberían tomar como evidencia suficiente para concluir una alteración del procesamiento temporal. A pesar de que esta prueba es mucho más difundida que su antecesora, es difícil encontrar información en relación con su sensibilidad y especificidad. En Brasil, se ha estimado una sensibilidad del $48 \%{ }^{33}$ en población pediátrica con trastornos del procesamiento auditivo, teniendo importantes variaciones de acuerdo con la edad. En población de adultos mayores, se ha estimado una sensibilidad de $88,6 \%$ y una especificidad de $8,9 \%{ }^{35}$.

\section{Prueba de gaps en ruido}

Una herramienta creada recientemente por Musiek y cols ${ }^{36}$ para evaluar la resolución auditiva temporal es la prueba de gaps en ruido o también conocida como GIN (del inglés gaps in noise). La prueba tiene una duración aproximada de 20 minutos y está conformada por una pista de 10 items iniciales de práctica y por cuatro pistas de prueba. Cada una de ellas contiene una serie de segmentos de ruido de banda ancha de 6 segundos de duración con una separación de 5 segundos entre cada uno de ellos. Cada segmento de ruido contiene de cero a tres intervalos de silencio, los que pueden tener una duración de 2, 3, 4, 5, 6, 8, 10, 12, 15020 $\mathrm{ms}$. En cada una de las pistas de prueba se repiten seis veces cada uno de los intervalos de silencio. La tarea solicitada al sujeto evaluado es presionar el pulsador del audiómetro cada vez que detecte la presencia de un intervalo de silencio. Solamente es necesario aplicar una pista de prueba para cada oído, quedando disponibles dos pistas más para realizar una reevaluación. Esto constituiría una gran diferencia en relación con el AFT-R y al RGDT, cuya presentación es siempre en una condición binaural. Al igual que la mayoría de las pruebas conductuales de procesamiento auditivo, se recomienda una intensidad de aplicación de 50 dB SL.

Para el análisis de esta prueba, se pueden utilizar dos parámetros distintos ${ }^{37}$ : el GDT aproximado y el puntaje (o porcentaje) final de items correctos. El GDT aproximado, abreviado también como "A.th" (del inglés approximate threshold), se define como la duración del intervalo de silencio más corto que el sujeto haya podido detectar en al menos cuatro de las seis presentaciones. Este nivel de rendimiento debería mantenerse o mejorar para los intervalos de mayor duración para que se considere el GDT aproximado del sujeto. En caso contrario, se debe buscar el próximo intervalo de silencio que cumpla con el criterio de detección de al menos cuatro de seis presentaciones. Por otro lado, el puntaje final de items correctos se refiere a la cantidad total de intervalos de silencios detectados por el sujeto. De esta manera, al haber diez duraciones posibles de intervalos de silencio, y cada uno se repite seis veces, el sujeto debería detectar 60 intervalos o gaps distintos para tener toda la prueba correcta. Este puntaje final se puede consignar en términos absolutos (por ejemplo, 42 gaps detectados del total de 60) 0 en términos de porcentaje (70\% para el mismo ejemplo).

Probablemente, el GIN es una de las pruebas conductuales de procesamiento auditivo que cuenta con la mayor cantidad de documentación en relación con su eficacia. Al interpretar los resultados utilizando el GDT aproximado, los autores del GIN ${ }^{36}$ han recomendado aplicar un criterio de corte de 5 
ms. De esta manera, la prueba alcanzaría una sensibilidad de $73 \%$ y una especificidad de $84 \%$, con un área bajo la curva ROC (receiver operator curve) de $0,87 \%$. Si los resultados son interpretados con el puntaje total, se ha sugerido la utilización de un puntaje de corte de 37 respuestas correctas (0 $62 \%$ ) para una sensibilidad del $56 \%$ y una especificidad del $87 \%$, con un área bajo la curva del 0,75 . Teniendo en cuenta el área bajo la curva, ambas formas de interpretación de la prueba serían considerados como adecuadas para determinar la presencia 0 ausencia de alteraciones en el SNAC y trastornos del procesamiento auditivo. Cabe señalar es posible mejorar aún más la sensibilidad de la prueba utilizando otros criterios de corte para el GDT aproximado, sin embargo, la especificidad de la prueba disminuiría significativamente. Dichos resultados demuestran que el GIN es una prueba sensible para determinar los trastornos del procesamiento auditivo.

\section{Consideraciones clínicas de las pruebas de resolución temporal}

En la actualidad, no existe claridad sobre la influencia que las pérdidas auditivas periféricas puedan tener sobre las pruebas conductuales de resolución temporal. En un estudio realizado por Matos y Frotas ${ }^{38}$, se compararon tres grupos de sujetos adultos sin pérdida auditiva, hipoacusia sensorioneural leve e hipoacusia sensorioneural moderada. Utilizando la prueba GIN, no se encontraron diferencias significativas entre los grupos, ni en el GDT aproximado ni el puntaje total de la prueba. Estos autores mencionaron incluso que la edad sería un factor más importante que la pérdida auditiva periférica. En otro estudio, se utilizó el RGDT para comparar dos grupos de sujetos adultos mayores con y sin pérdida auditiva sensorioneural de leve a moderadamente severa ${ }^{39}$. En este estudio tampoco se encontraron diferencias significativas entre los grupos estudiados.

De manera contradictoria, en un estudio realizado por Lima y Miranda ${ }^{40}$, se demostró que existen diferencias significativas en el resultado de la prueba GIN entre adultos mayores con audición periférica normal y con hipoacusia sensorioneural leve a moderada, teniendo el grupo con pérdida auditiva un desempeño más bajo. Incluso, se ha reportado que aquellos sujetos con hipoacusia unilateral tendrían resultados significativamente peores que aquellos con audición normal en tareas de detección de gaps ${ }^{41}$. Otros autores han evidenciado mayores dificultades en este tipo de tareas cuando la hipoacusia se encuentra en el oído derecho, en relación con aquellos que presentan hipoacusia unilateral de oído izquierdo $0^{42}$.

No solo las hipoacusias sensorioneurales tendrían un efecto sobre las pruebas de resolución temporal. Moore y cols ${ }^{43}$ describieron que las hipoacusias de conducción también podrían generar alteraciones en el procesamiento auditivo, puntualmente en la resolución temporal. En un grupo de niños con hipoacusia de conducción unilaterales y bilaterales, se evidenció un rendimiento deficiente en la prueba RGDT en relación con un grupo de niños controles sin hipoacusia, obteniendo incluso un perfil similar a un tercer grupo de menores diagnosticados con trastornos del procesamiento auditivo ${ }^{44}$. Más recientemente, se comparó el procesamiento temporal a través de la prueba GIN en un grupo de adultos con audición normal y otro grupo con hipoacusia de conducción bilateral de leve a moderada ${ }^{45}$, encontrando diferencias significativas en relación con el porcentaje de respuestas correctas, así como en el GDT aproximado. En una revisión anterior ${ }^{10}$, se ha mencionado que algunas pruebas de procesamiento temporal como la prueba de patrones de frecuencia y de duración, son resistentes a pérdidas auditivas periféricas de grado leve y moderado. Esto parece indicar que algunos procesos centrales como el ordenamiento auditivo temporal, no se verían afectado por pérdidas auditivas periféricas. Por el contrario, la resolución temporal parece depender en gran medida de un adecuado funcionamiento de todo el sistema auditivo y parece ser sensible a periodos relativamente cortos de deprivación auditiva ${ }^{43}$. Esto se ha podido evidenciar en estudios de menores con historial de otitis media con efusión quienes, a pesar de no cursar con la patología al momento de las evaluaciones de procesamiento temporal, han demostrado tener un rendimiento significativamente menor a otros menores sin antecedentes de patología de oído medio ${ }^{46}$.

Otro tema controversial en relación con las pruebas conductuales de resolución temporal es la ventaja que tendría un oído sobre otro. Utilizando 
la prueba GIN en sujetos normales, se ha documentado un GDT similar para ambos oídos, independiente del oído en el cual se inició la prueba ${ }^{34,47}$. Este hallazgo ha sido descrito también en un grupo de sujetos con trastorno del procesamiento auditivo utilizando la misma prueba ${ }^{36}$. Sin embargo, otro estudio ha informado una ventaja significativa para el GDT del oído derecho en comparación con el oído izquierdo $0^{42}$. Los autores concluyeron que ambos oídos tendrían distintos grados de desempeño de procesamiento temporal, lo que se podría deber a la especialización de los hemisferios cerebrales ${ }^{41}$. En general, la corteza auditiva del hemisferio izquierdo está especializada en el procesamiento de estímulos acústicos con estructura temporal compleja (como el habla), el hemisferio derecho es importante en el procesamiento espectral y favorece los componentes suprasegmentales ${ }^{42}$. La asimetría entre ambos hemisferios beneficiaría el procesamiento de cierto tipo de estímulos cuando son presentados al oído contralateral ${ }^{41,48}$. A pesar de que existe literatura contradictoria al respecto, las pruebas de detección de gaps se consideran especialmente sensibles a lesiones corticales de hemisferio izquierdo².

En términos generales, la literatura indicaría que no existen diferencias entre hombres y mujeres en las pruebas de resolución temporal. Se ha reportado que tanto para las pruebas RGDT como GIN, no existirían diferencias significativas, al menos en un grupo de niños de 6 a 14 años ${ }^{34}$. Los estudios iniciales de la prueba GIN indican que tampoco existirían diferencias por género en sujetos con alteraciones del procesamiento auditiv $0^{36}$. Sólo un estudio ${ }^{49}$ ha reportado un rendimiento significativamente mayor en hombres utilizando el RGDT y el GIN. Sin embargo, los autores destacan que esto pudo deberse a que el grupo de sujetos de sexo masculino eran estudiantes de un curso de musicoterapia. Este sería un detalle importante debido a que la resolución temporal se trataría de un proceso altamente entrenable. Algunos estudios han demostrado una mayor habilidad de resolución temporal en músicos en comparación con sujetos sin entrenamiento musical ${ }^{50,51}$.

Un aspecto que considerar durante la aplicación e interpretación de las pruebas de resolución temporal es que parecieran estar influenciadas por el nivel educacional de los sujetos. En un estudio realizado por Lima y Miranda ${ }^{40}$ se observó que, a mayor escolaridad de los sujetos evaluados, menores fueron los GDT aproximados. Los autores plantearon que estos resultados se deben al efecto que la educación tendría en la interacción de las capacidades inferenciales con otras funciones cognitivas como la memoria de trabajo, las capacidades léxico-semánticas y el conocimiento del mundo. Relacionado a esto, se ha evidenciado que el nivel socioeconómico también sería un factor que considerar en la interpretación de estas pruebas. Utilizando el RGDT y el GIN, Balen y cols ${ }^{52}$ compararon tres grupos de niños de estrato socioeconómico alto, medio y bajo, encontrando diferencias significativas entre los grupos. Esto sería particularmente importante ya que al obtener valores normativos de estas pruebas es fundamental considerar, al igual que en pruebas neuropsicológicas, el nivel de escolaridad y la influencia del nivel socioeconómico.

A pesar de que sería esperable encontrar resultados similares en las tres pruebas de resolución temporal, se han reportado diferencias en los resultados tanto en sujetos con trastorno del procesamiento auditivo como en sujetos normales. Un ejemplo de esto es el estudio realizado por Chermak y $L e^{30}$, en el cual se comparó el rendimiento del AFT-R, RGDT y el GIN en un grupo de niños normales (se incluyó además una cuarta prueba, pero no es considerada en la presente revisión). En dicho estudio se encontraron diferencias significativas entre los promedios del umbral de fusión auditiva (AFT-R), el GDT (RGDT) y el GDT aproximado (GIN). Estas diferencias han sido atribuidas a la tarea que debe realizar el sujeto evaluado, al tipo de estímulo utilizado y a la forma de presentación de éste. En otra investigación se comparó el RGDT y el GIN en sujetos músicos adultos, niños con trastorno del procesamiento auditivo y adultos con brotes psicóticos, encontrando que los promedios del GDT aproximado del GIN y las desviaciones estándar fueron significativamente menores en relación con el GDT del RGDT ${ }^{53}$. Los autores de dicho trabajo propusieron que la falta de correlación entre ambas pruebas se podría deber a que estarían evaluando procesos diferentes. A conclusiones similares llegaron Balen y cols tras estudiar un grupo de niños normales con el RGDT y el GIN34.

En la literatura, se han encontrado pocos registros publicados en los que se haya utilizado 
alguna prueba clínica de resolución temporal asistida de audífonos o implante coclear. Uno de los artículos que detalla este procedimiento, es el de Duarte y cols $^{54}$, quienes utilizaron la prueba GIN para evaluar la resolución temporal en grupo de adultos usuarios de implante coclear. La prueba se realizó utilizando un sistema de campo libre de dos parlantes ubicados a un metro de los sujetos evaluados a $0^{\circ}$ azimuth en el plano vertical y horizontal. Dentro de los hallazgos destaca el hecho de que el grupo de usuarios de implante presenta un rendimiento significativamente menor a otro grupo de adultos de audición normal. Esto fue atribuido a las limitaciones propias del implante coclear en entregar la suficiente información temporal como para realizar este tipo de tareas.

Por último, cabe destacar que a nivel nacional se han publicado dos estudios en relación con la resolución temporal. La finalidad del primero de ellos fue establecer valores referenciales para la prueba RGDT $^{55}$. En la obtención de éstos participaron 40 sujetos de entre 18 y 50 años. Como resultado, al igual que en otras investigaciones mencionadas previamente, no se evidenciaron diferencias entre géneros y se obtuvo una correlación significativa entre las subpruebas frecuenciales. Asimismo, se observó que las puntuaciones obtenidas no difieren de aquellas reportadas en sujetos hablantes de lengua inglesa, quedando el percentil 10 como puntaje de corte en $15 \mathrm{~ms}$. Un gran aporte de esta publicación es que para la subprueba que utiliza tonos clic establece el percentil 10 como criterio de corte en $10 \mathrm{~ms}$, incorporando por primera vez un puntaje de referencia para este item. En un segundo trabajo nacional, se estudió la resolución temporal a través de la prueba RGDT en un grupo de 30 adultos mayores entre 60 y 85 años $^{56}$. En términos generales, se evidenciaron GDT más elevados que los establecidos como referencia en el estudio anterior ${ }^{55}$. Además, se realizó una comparación entre aquellos sujetos con niveles de audición acorde a su edad según el estándar ISO 7029 y aquellos que presentaban umbrales auditivos más elevados de lo esperado. Se evidenció en este último grupo GDT, significativamente mayores. Otro hallazgo interesante es que los GDT para las frecuencias 2.000 y $4.000 \mathrm{~Hz}$, se correlacionaron de manera inversa con los porcentajes de reconocimiento verbal de monosílabos (a mayores GDT, menor fue el porcentaje). Estas dos observaciones sugieren en primer lugar, al igual que algunos estudios internacionales ${ }^{40-42}$, que se debe cautelar la interpretación de las pruebas de resolución temporal en presencia de hipoacusias sensorioneurales. En segundo lugar, la correlación de los GDT y el desempeño en una prueba de reconocimiento verbal sugiere que la habilidad de resolución temporal estaría influenciando otros procesos auditivos más complejos, como puede ser la discriminación, identificación y reconocimiento de palabras.

\section{CONCLUSIONES}

Los aspectos temporales de la audición son considerados la base para el adecuado funcionamiento del resto de los procesos auditivos centrales. Dentro de ellos, la resolución temporal es uno de los más ampliamente estudiados por su relevancia en la percepción de los aspectos más sutiles del lenguaje y en la discriminación de los sonidos del habla. A diferencia de otros procesos auditivos, la resolución temporal ha demostrado ser sensible a periodos de tiempo relativamente cortos de deprivación auditiva. Esto sería de suma importancia por dos razones. En primer lugar, la interpretación de un rendimiento deficiente en las pruebas de resolución temporal en un sujeto con hipoacusia no puede ser atribuido únicamente a aspectos relacionados con la intensidad de presentación de la prueba 0 a aspectos periféricos de la audición. Debe considerarse cualquier tipo de hipoacusia, incluso aquellas unilaterales, como factores significativos de deprivación que pueden afectar esta habilidad temporal. En segundo lugar, debería considerarse la evaluación de la resolución temporal en aquellos casos que, a pesar de haber resuelto la pérdida auditiva periférica, se mantengan algunas dificultades perceptuales 0 quejas subjetivas de audición. No está claro si estas secuelas en la resolución temporal se resuelven de manera espontánea 0 se prolongan de manera indefinida en el tiempo.

En la actualidad, sólo existen pruebas conductuales aplicables en la clínica de procesamiento temporal limitadas al ordenamiento y a la resolución temporal. La utilización de las pruebas de resolución temporal descritas en el presente do- 
cumento, en conjunto con las pruebas de patrones de frecuencia y duración, permiten una evaluación exhaustiva, rápida y sencilla de la capacidad del sistema auditivo para procesar el sonido en una ventana temporal definida. EI GIN es la prueba que cuenta con mayor cantidad de reportes sobre sensibilidad y especificidad frente a lesiones del SNAC, por lo que se recomienda su incorporación a la batería de evaluación conductual del procesamiento auditivo. Sin embargo, tanto los resultados del AFT-R, RGDT como del GIN, deben analizarse con especial cautela, considerando que están fuer-

\section{BIBLIOGRAFÍA}

1. American Speech-Language-Hearing Association. American Speech-Language-Hearing Association. [Online].; 2005. Available from: http://www.asha.org/policy/TR2005-00043.htm.

2. BeLLIS T. Assessment and Management of Central Auditory Processing Disorders in the Educational Setting: From Science to Practice. Segunda ed. San Diego, CA: Plural Publishing; 2011.

3. Chermak G, Musiek F. Central auditory processing disorders: New perspectives San Diego, CA: Singular; 1997.

4. Brooke J. Temporal Processing Test. In Musiek F, Chermak G. Handbook of Central Auditory Processing Disorder: Auditory Neuroscience and Diagnosis. Segunda ed. San Diego, CA: Plural Publishing; 2014. p. 405-34.

5. Musiek F, Chermak G. Psychophysical and behavioral peripheral and central auditory tests. In: Aminoff M, Boller F, Swaab D. Handbook of Clinical Neurology: The Human Auditory System Fundamental Organization and Clinical Disorders. Waltham, MA: Elsevier B.V.; 2015. p313-32.

6. Gelfand S. Hearing: An introduction to psychological and physiological acoustics. Quinta ed. London, UK: Informa Healthcare; 2010.

7. GreEn D. Temporal auditory acuity. Psychol Rev 1971; 78: 540-51.

8. Phillips D. Auditory gap detection, perceptual channels and temporal resolution in speech perception. J Am Acad Audiol 1999; 10: 343-54.

9. Eggermont J. Neural responses in primary auditory cortex mimic psychophysical, across- temente influenciados por la edad de los sujetos evaluados, nivel de escolaridad, nivel socioeconómico y entrenamiento auditivo previo. Además, se debe tener presente que a pesar de que las pruebas utilicen metodologías y paradigmas de evaluación similares, la poca coherencia entre sus resultados indicaría que estarían evaluando distintos subprocesos, por lo que no deben considerarse como pruebas homologables. Por último, se recomienda establecer valores normativos locales teniendo en cuenta las variables reportadas en la literatura que podrían influenciar los resultados.

frequency-channel, gap-detection thresholds. $J$ Neurophysiol 2000; 84: 1453-63.

10. Marcotti A, Rivera S. Prueba de patrones de frecuencia y patrones de duración: Evaluación del ordenamiento auditivo temporal. Rev Otorrinolaringol Cir Cabeza Cuello 2017; 77: 339-50.

11. Steinschneider M, Volkov I, Noh M, Garell P, HowaRd M. Temporal encoding of the voice onset time phonetic parameter by field potentials recorded directly from human auditory cortex. $J$ Neurophysiol 1999; 82: 2346-57.

12. Roldán Y, Soto-Barba J. El V.O.T. de /p-t-k/ y $/ b-d-g /$ en el español de Valdivia: un análisis acústico. Estud Filol 1997; 32: 27-33.

13. Belinchón M, Riviere A. Psicología del lenguaje: investigación y teoría. Sexta ed. Madrid: Trotta; 2009.

14. CePEDA G. El valor contrastivo de /p/y /b/a través de tres indicadores. Estud filol 1989; 24: 11-8.

15. Soto-Barba J. ¿Los fonemas /b/ y /p/ se diferencian por la sonoridad? Estud filol 1994; 29: 33-8.

16. Soto-Barba J, Valdivieso H. Caracterización fonético-acústica de la serie de consonantes /p-t-k/ vs. /b-d-g/. Onomazein 1999; 4: 125-33.

17. Hurley R, Hurley A. Psychoacoustic considerations and implications for the diagnosis of central auditory processing disorder. In Musiek F, Chermak G. Handbook of Central Auditory Processing Disorder: Auditory Neuroscience and Diagnosis. Segunda ed. San Diego, CA: Plural Publishing; 2014. p17-58.

18. KeITH B. Random Gap Detection Test St. Louis, MO: Auditec Inc; 2000. 
19. Eggermont J. Neural correlates of gap detection and auditory fusion in cat auditory cortex. Neuroreport 1995; 6: 1645-8.

20. Purcell D, John S, Schneider B, Picton T. Human temporal auditory acuity as assessed by envelope following responses. J Acoust Soc Am 2004; 116: 3581-93.

21. Formby C, Forrest T. Detection of silent temporal gaps in sinusoidal markers. $J$ Acoust Soc Am 1991; 89: 830-7.

22. Heinrich A, Alain C, Schneider B. Within- and between-channel gap detection in the human auditory cortex. Neuroreport 2004; 15: 2051-6.

23. Phillips D, Hall S. Independence of frequency channels in auditory temporal gap detection. $J$ Acoust Soc Am 2000; 108: 2957-63.

24. Phillips D, Taylor T, Hall S, Carr M, Mossop J. Detection of silent intervals between noises activating different perceptual channels: some properties of "central" auditory gap detection. J Acoust Soc Am 1997; 101: 3694-705.

25. Formby C, Gerber M, Sherlock L, Magder L. Evidence for an across-frequency, between-channel process in asymptotic monaural temporal gap detection. $J$ Acoust Soc Am 1998; 103: 3554-60.

26. LISTER J, BEsing J, KOEHNKE J. Effects of age and frequency disparity on gap discrimination. $J$ Acoust Soc Am 2002; 111: 2793-800.

27. LISTER J, KoEHNKE J, BesING J. Binaural gap duration discrimination in listeners with impaired hearing and normal hearing. Ear Hear 2000; 21: 141-50.

28. Grose J, Hall J, Buss E, Hatch D. Gap detection for similar and dissimilar gap markers. J Acoust Soc Am 2001; 109: 1587-95.

29. McCroskey R, Keith R. Auditory fusion testrevised: Instruction and user's manual St. Louis, MO: Auditec Inc; 1996.

30. Chermak G, Lee J. Comparison of children's performance on four tests of temporal resolution. J Am Acad Audiol 2005; 16: 554-63.

31. McCroskey R, Kidder H. Auditory fusion among learning disabled, reading disabled, and normal children. J Learn Disabil 1980; 13: 69-76.

32. Friberg J, McNamara T. Evaluating the reliability and validity of (Central) Auditory Processing Tests: A preliminary investigation. J Educ Audiol 2010; 16: 4-17.

33. Dias K, Jutras B, Acrani I, Pereira L. Random Gap Detection Test (RGDT) performance of individuals with central auditory processing disorders from 5 to 25 years of age. Int J Pediatr Otorhinolaryngol 2012; 76: 174-8.

34. Balen S, Liebel G, Boeno M, Mottecy C. Resolução temporal de crianças escolares. Rev CEFAC 2009; 11: 52-61.

35. Vellozo F, Schwantes A, Souza A, Peixe B, Biaggio E, MaRtins $Q$, ET AL. Temporal resolution in elderly. Rev CEFAC 2016; 18: 355-61.

36. Musiek F, Shinn J, Jirsa R, Bamiou D, Baran J, ZAIDA E. GIN (Gaps-In-Noise) test performance in subjects with confirmed central auditory nervous system involvement. Ear Hear 2005; 26: 608-18.

37. Shinn J, Chermak G, Musiek F. GIN (Gaps-In-Noise) performance in the pediatric population. $\mathrm{JAm}$ Acad Audiol 2009; 20: 229-38.

38. Matos G, Frotas S. Resolução temporal em perdas auditivas sensorioneurais. Audiol Commun Res 2013; 18: 30-6.

39. Azzolini V, Ferreira M. Temporal Auditory Processing in Elders. Int Arch Otorhinolaryngol 2010; 14: 95-102.

40. Lima I, Miranda-Gonsalez E. Effects of age, schooling and hearing loss on temporal processing in elderly. Rev CEFAC 2016; 18: 33-9.

41. SinINGER Y, DE BODE S. Asymmetry of temporal processing in listeners with normal hearing and unilaterally deaf subjects. Ear Hear 2008; 29 : 228-38.

42. Nishihata R, Vieira M, Pereira L, Chiari B. Temporal processing, localization and auditory closure in individuals with unilateral hearing loss. Rev Soc Bras Fonoaudiol 2012; 17: 266-73.

43. Moore D, Hartley D, Hogan S. Effects of otitis media with effusion (OME) on central auditory function. Int J Pediatr Otorhinolaryngol 2003; 67: S63-67.

44. Balen S, Bretzke L, Mottecy C, Liebel G, Boeno M, Gondim L. Temporal resolution in children: comparing normal hearing, conductive hearing loss and auditory processing disorder. Braz $J$ Otorhinolaryngol 2009; 75: 123-9.

45. Bayat A, Farhadi M, Emamdjomeh $H$, Saki $N$, Mirmomenı G, Rahim F. Effect of conductive hearing loss on central auditory function. Braz J Otorhinolaryngol 2017; 83: 137-41.

46. Khavarghazalanı B, Farahani F, Emadi M, Hossenı Z. Auditory processing abilities in children with chronic otitis media with effusion. Acta Otolaryngol 2016; 136: 456-9. 
47. Samelli A, Schochat E. Estudo da vantagem da orelha direita em teste de detecção de gap. Rev Bras Otorrinolaringol 2008; 74: 235-40.

48. Firszt J, Ulmer J, GaggL W. Differential representation of speech sounds in the human cerebral hemispheres. Anat Rec A Discov Mol Cell Evol Biol 2006; 288: 345-57.

49. Zaidan E, Garcia A, Tedesco M, Baran J. Desempenho de adultos jovens normais em dois testes de resolução temporal. Pró-Fono $R$ Atual Cient 2008; 20: 19-24.

50. Kumar P, Sanju H, NikHil J. Temporal Resolution and Active Auditory Discrimination Skill in Vocal Musicians. Int Arch Otorhinolaryngol 2016; 20: 310-4.

51. Sangamanatha A, Fernandes J, Bhat J, Srivastava M, PrakRITHI S. Temporal resolution in individuals with and without musical training. J Ind Sp Hear Assoc 2012; 26: 27-35.

52. Balen S, Boeno M, Liebel G. A influência do nível socioeconômico na resolução temporal em escolares. Rev Soc Bras Fonoaudiol 2010; 15: 7-13.

53. Iliadou V, Bamiou D, Chermak G, Nimatoudis I. Comparison of two tests of auditory temporal resolution in children with central auditory processing disorder, adults with psychosis, and adult professional musicians. Int J Audiol 2014; 53: 507-13.

54. Duarte M, Gresele A, Pinheiro M. Temporal processing in postlingual adult users of cochlear implant. Braz J Otorhinolaryngol 2016; 82: 304-9.

55. Fuente A, McPherson B. Normalización de la prueba de "Random gap detection" para poblaciones nativas hispanoparlantes. Revista Chilena de Fonoaudiología 2005; 6: 19-28.

56. Fuente A, Hormazábal X. Evaluación de la discriminación del habla y resolución temporal en un grupo de adultos mayores hispanohablantes. Revista Chilena de Fonoaudiología 2007; 8: 35-55.

Correspondencia: Anthony Marcotti F. 